\title{
Research on the Cooperative Dispatch of Electric Vehicle and Distributed Power Sources
}

\author{
Shijie Wang \\ School of Electrical \& Electronic Engineering, North China Electric Power University, Baoding \\ 071003 China
}

Keywords: vehicle to grid (V2G); distributed power sources; cooperative scheduling control.

\begin{abstract}
The rapid application of electric vehicle (EV) is a trend in recent years. Meanwhile, it will have a significant impact on power system. In order to reducing the disadvantages of EV accessing to the grid and maximizing the benefits of such controllable load, the scheduling control strategy of EV is becoming a hot spot of research nowadays. This paper focuses on research achievements combining new energy and EV, such as the construction of photovoltaic (PV) converter (charging) station, the cooperative dispatch of EV connecting to the grid (Vehicle to Grid, V2G) as distributed power source and wind generation, PV power generation, fuel cells and other distributed powers. Particularly, this paper wholly describes the related research on optimal dispatch of V2G and other distributed power sources in the micro grid. Finally, it does some forecasting of the development trend of EV.
\end{abstract}

\section{Introduction}

In the past few years, due to advances in electric vehicle technology, particularly the development of the higher storage density battery and better charging performance motor and its control system makes the development of EVs significantly faster. More importantly, in many countries, especially in some developing countries such as China, the auto industry is developing very quickly, sustained and rapid increase in the number of cars, leading to rising oil consumption, air pollution and environmental deterioration and natural disasters caused by greenhouse gases tend to be frequent and severe over the past ten years. Against this background, many countries adopted different energy policies in order to enhance economic efficiency, energy security and reduce environmental pollution. The EVs have had a rapid development which is promoted by such policies, technologies and environments as well as the following advantages [1].

Enhance economic efficiency: In some developed counties such as Australia, the EV consumes about one third of the cost of fuel vehicle per kilometer on average. Therefore, if the investment cost structure of an EV is reasonable, then the owner will have apparent cost savings. It should be noted that EVs better adapt to the existing power facilities and need less additional investment when compared with other new energy vehicles such as hydrogen fuel vehicles and so on.

Contribute to energy security: Currently, transportation almost entirely depends on oil (and gas), resulting in the price of oil (and gas) being high and much volatile. It may lead to a series of serious energy security issues in China since the storage of fossil energy is not sufficient enough. In addition, there is no alternative which can be widely used to replace petroleum.

Reduce the impact on the environment: Emissions of conventional fuel (and gas) vehicles account for a large proportion of greenhouse gas emissions. The widespread adoption of EVs, can reduce not only regional air pollution caused by traditional fuel vehicles in densely populated areas, but also the overall greenhouse gas emissions.

New energy is an outstanding representative which is related to environmental protection and low-carbon economy. It is a research hot spot that how to maximize the use and consumption of new energy in the grid in recent years. The V2G technology will brings much more convenience to researchers of how to exploit new energy, which is a revolutionary chance for the grid. 


\section{The cooperative dispatch control mode of new energy and EV}

The growth of EV is an important way which is established generally to ensure energy security and low-carbon economy in transition. It is inseparable for scale application of EV to build up enough basic charging facilities. According to China's current structure of primary energy, the backstairs carbon emissions caused by EVs being connected to the gird directly will not be less than fuel vehicles [2].

The construction of PV converter station combining the technology of PV generating and the demand of EVs charging can efficiently improve the utilization of cleaning energy and environmental benefits. For wind which occupies a high proportion in renewable energy, the cooperative scheduling including EV and other distributed energy can smooth load curve and enhance the ability of accepting EV to the grid. Thus, distributed energy and EV have a close relationship.

\section{Photovoltaic power generation and electric vehicle}

Speeding up the construction of PV converter station is a typically integrated way for EV to use renewable energy locally. The key problem is about the power distribution and optimal scheduling. Paper [3] proposed a dynamic power allocating approach for PV converter station which accounts for the storage of electricity. This approach contains two aspects. On the one hand, it should add the results of short-term forecasts for solar-electricity demand to build up the solar-electricity service model. On the other hand, it should add the total amount of available power battery of the station every time to establish power allocating model.

It verifies the correctness of the model from balancing power of the system, analyzing operating performance and other aspects of rationality through simulating the practical operating examples of PV converter station. The results of simulations prove that this approach efficiently increases the proportion of the local utilization of solar under the circumstance of ensuring availability of solar-electricity service.

Paper [4] proposes a multi-objective optimal dispatching model which contains the cost of purchasing electricity and circulate electricity of battery. The optimal result of this model embodies apparent advantages after being compared with other charging plans.

Another key problem is the capacity allocation of PV converter station. Paper [5] establishes a system capacity optimal allocation model as the target of maximizing the annual profit of PV converter station when it takes the echelon use of power battery and the deployment of PV converter station into account.

To confirm the annual echelon using capacity of power battery, it establishes the echelon using capacity calculating model based on Monte Carlo simulation refer to capacity degradation model and statistical model of EV's daily mileage.

Paper [6] represents a multi-objective capacity optimization allocation mathematics model which considers renewable energy availability and investing operation costs in order to combining the maximization of renewable energy availability and the minimization of system investment and prime cost of operation.

\section{Wind generation and electric vehicle}

The reasonable cooperation of EV and wind could enhance the degree of transforming wind into electricity. Meanwhile, it makes huge influence to the operation status of power system that the charging of EV and the randomness and time variability of wind. Paper [7] builds up dynamic probability model about EV charging, wind generation and basic load based on the coefficient of variation.

The results of simulation represent that it can gain comprehensive current distribution. This paper[8] establishes multi-time scale EV-wind cooperative scheduling mathematical model, and analyses the practicability of dispatching EV charging in order to smoothing equivalent load fluctuations in power system and consuming excessive wind power during night. Based on the above aspects, paper [9] proposes cooperative dispatch measurement model of carbon emission reduction benefits, and proves that cooperative dispatch is an effective approach to the scale of coordinate utilization of wind and EV. Paper [10] introduces the up-to -date development of project EDOSON in 
power system of Denmark which contains extensive wind generation and EVs, that is, the management which is adopted technology of virtual power plant to realize intelligent charging and using electricity of EV. It represents natural cases of consuming wind generation.

\section{Modes of micro-grid}

PV and wind power are typical distributed energies. Micro-grid has an inherent advantage in integration of distributed power. The EVs can be taken as the "move" distributed power through V2G technology.

And PV plants can be taken as an ultra-small micro-grid. The PV cells, wind turbines, fuel cells, diesel generators, electric cars and other series of distributed power are able to be accepted by micro-grid. Meanwhile, to avoid the "curse of dimensionality" problem in optimization scheduling of large-scale of EVs and the negative impact of distributed energies and EVs, considering the guidance issued by the State Grid Corporation, the problem can be taken as the optimization scheduling problem with EVs and power exchange plants through the use of hierarchical partitioning operation and management structure.

Paper [11] simulates the charging and discharging behavior of EVs with the method of the Monte Carlo simulation and the demand characteristics of EVs when charging disorderly as well as the capacity curve when charging orderly in network are analyzed. The multi-objective economic dispatch model of micro-grid considering EVs is built.

But the ordered charge is not equivalent to the result of intelligent scheduling. Paper [12] proposes an intelligent calculation model based on adaptive charging and discharging of EVs in micro-grid. The calculation model presents the control strategy of adaptive charging and discharging of EVs and the key devices are designed. The technology to meet the adaptive electric energy interaction between the EVs and intelligent micro-grid is studied.

Paper [10] studies the operation strategy of power exchange plants of EVs in the environment of micro-grid. The battery scheduling processes of power exchange plants is formulated to maximize scheduling signal in response to micro-grid.

\section{Conclusion}

With the vigorously promotion of study of EVs in different countries, the EV industry witnesses a huge development. The charging and discharging behavior of EVs will have a major impact on the grid. This paper focuses on the research on the collaborative utilization of EVs and new energy. The problems of grid brought by the EVs are described. The smart charging strategies are summarized and the suggestions and prospects are presented.

There are more and more researches on EVs which not only focus on energy, environment and key technology, but also on economic evaluation and management system. In our country, the future development of EVs should consider the facts in scientific management, comprehensive decision-making and so on. The centralized scheduling and micro-grid dispatching should be taken into account at the same time to promote the development of China's electric vehicle industry, improve grid scheduling control level and reach the purpose of a strong smart grid.

\section{Reference}

[1] Z. C. Hu, Y. H. Song, Z. W. Xu, Z. W. Luo, K. Q. Zhan and L. Jia: CSEE,(2012)No.4,p.1-10

[2] J. Ma, C. N. Nian: Agricultural Equipment \& Vehicle Engineering, (2011) No.2

[3] Z. Chen, N. Liu, X. Y. Lu, X. N. Xiao, J. H. Zhang: China Electro technical Society, (2014) No.4, p. 306-315

[4] X. Y. Lu, N. Liu, Z. Chen, J. H. Zhang, X. N. Xiao, :China Electro technical Society, (2014) No.8, p. $46-56$

[5] N. Liu, X. Tang, S. Duan, J. H. Zhang: CSEE, (2013) No.4, p. 34-44, 6 
[6] Z. Chen, X. N. Xiao, X. Y. Lu, N. Liu, J. H. Zhang: China Electro technical Society, (2013) No.6, p. 238-248

[7] D. F. Cai, B. Qian, J. F. Chen, M. Q. Yao: Grid Technology, (2013) No.3, p. 590-596

[8] L.H. Yang, S. Xu: Automation of Electric Power Systems, (2011) No.14, p.43-47

[9] D.Y. Yu, S.G. Song, B. Zhang, X.S. Han: Automation of Electric Power Systems, (2011) No.14, p. 24-29

[10] J.H. Zhao, F.S. Wen, Y.S, Xue, C.Y. Dong, J.B. Xin: Automation of Electric Power Systems, (2010) No.20, p. 22-29

[11] D.Y. Yu, H.L. Huang, M. Lei, X. Li, B. Zhang, X.S. Han: Automation of Electric Power Systems, (2012) No.10, p.14-18

[12] H.B. Wu, X.F. Hou, B. Zhao, C.Z. Zhu: Automation of Electric Power Systems, (2014) No.9, p. 77-84, 99. 